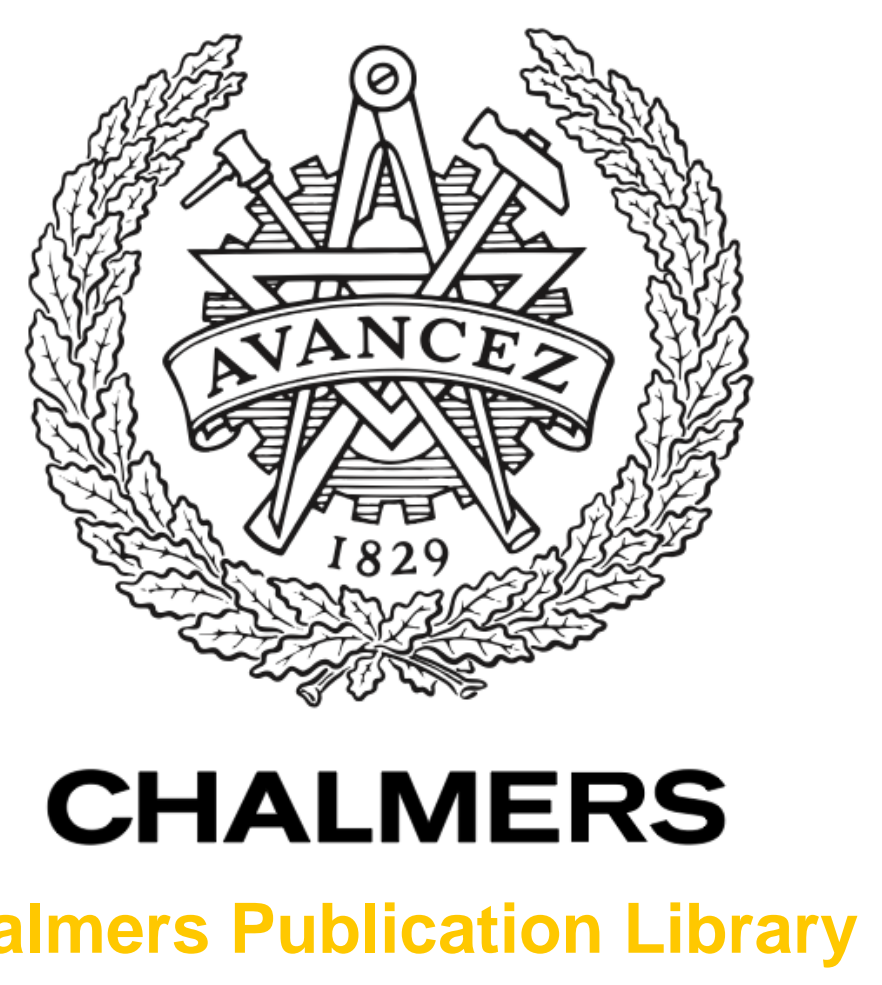

Challmers Publication Library

Moving Cells: A Promising Solution to Boost Performance for Vehicular Users

This document has been downloaded from Chalmers Publication Library (CPL). It is the author's version of a work that was accepted for publication in:

leee Communications Magazine (ISSN: 0163-6804)

Citation for the published paper:

Yutao, S. ; Vihriala, J. ; Papadogiannis, A. (2013) "Moving Cells: A Promising Solution to Boost Performance for Vehicular Users". Ieee Communications Magazine, vol. 51(6), pp. $62-68$.

http://dx.doi.org/10.1109/MCOM.2013.6525596

Downloaded from: http://publications.lib.chalmers.se/publication/181014

Notice: Changes introduced as a result of publishing processes such as copy-editing and formatting may not be reflected in this document. For a definitive version of this work, please refer to the published source. Please note that access to the published version might require a subscription.

Chalmers Publication Library (CPL) offers the possibility of retrieving research publications produced at Chalmers University of Technology. It covers all types of publications: articles, dissertations, licentiate theses, masters theses, conference papers, reports etc. Since 2006 it is the official tool for Chalmers official publication statistics. To ensure that Chalmers research results are disseminated as widely as possible, an Open Access Policy has been adopted.

The CPL service is administrated and maintained by Chalmers Library. 


\title{
Moving Cells: A Promising Solution to Boost Performance for Vehicular Users
}

\author{
Yutao Sui, Chalmers University of Technology \\ Jaakko Vihriälä, Nokia Siemens Networks \\ Agisilaos Papadogiannis, Chalmers University of Technology \\ Mikael Sternad, Uppsala University \\ Wei Yang and Tommy Svensson, Chalmers University of Technology
}

${ }^{1}$ Pico eNBs and RRHs need dedicated backhaul connections, whereas inband FRNs utilize the same carrier frequency as the UE to communicate with their donor eNBs. An important difference between a pico eNB and an $R R H$ is that a pico eNB is a low-power eNB that can form its own cells underlying the macro $e N B$, while an RRH is a part of the macrocell $\mathrm{eNB}$, and is deployed distributively to either extend coverage or improve local capacity. Home eNBs are deployed inside residential or official buildings in an unplanned way to form femtocells and offload the data traffic when users are at home or at work.

${ }^{2} A$ macro eNB is called $a$ donor eNB when it serves the backhaul links of RNs as well as UE within its coverage.

\begin{abstract}
In future wireless networks, a significant number of users accessing wireless broadband will be vehicular (i.e., in public transportation vehicles like buses, trams, or trains). The Third Generation Partnership Project has started to investigate how to serve these vehicular users cost-effectively, and several solutions have been proposed. One promising solution is to deploy a moving relay node (MRN), on a public transportation vehicle that forms its own cell inside the vehicle to serve vehicular users. By proper antenna placement, an MRN can reduce or even eliminate the vehicular penetration loss that affects communication. Moreover, MRNs can exploit various smart antenna techniques and advanced signal processing schemes, as they are less limited by size and power than regular user equipment. However, there are also challenges in using MRNs, such as designing efficient interference management techniques as well as proper mobility management schemes to exploit the benefit of group handovers for vehicular UE devices served by the same MRN. Nevertheless, initial system-level evaluation results indicate that a dedicated MRN deployment shows great potential to improve the vehicular user experience, and thereby can potentially bring significant benefits to future wireless communication systems.
\end{abstract}

\section{INTRODUCTION}

The Third Generation Partnership Project (3GPP) has standardized Long Term Evolution (LTE), which is aimed at improving the spectral efficiency of a cellular network. A more intelligent base station, the evolved Node B (eNB), was introduced to simplify the system architecture, and minimize the control and user plane latency. By employing advanced multi-antenna multiple-input multiple-output (MIMO) techniques, carrier aggregation (CA), and other schemes, the most recent LTE standard, LTE-
Advanced, can support a downlink speed of up to $1 \mathrm{~Gb} / \mathrm{s}[1]$.

The capacity offered by macro eNBs in the current LTE-Advanced system is not evenly distributed across their coverage areas; user equipment (UE) in the cell center can achieve much higher throughput than cell edge UE. The heterogeneous and small cell networks (HetSNets) solution addresses the capacity needs in certain data-intensive hotspot areas, and provides a ubiquitous user experience. An overview of a typical HetSNets scenario is illustrated in Fig. 1. Different types of low-power nodes, including pico eNBs, remote radio heads (RRHs), inband fixed relay nodes (FRNs), moving relay nodes (MRNs), and home eNBs, ${ }^{1}$ have been introduced. These low-power nodes are deployed underlying macro eNBs to either extend coverage or increase network capacity locally [2].

The number of users using wireless broadband services while riding in public transportation vehicles is growing rapidly, due to the high penetration of smart phones, tablets, and increasingly portable laptops [3]. In recent 3GPP studies, a dedicated MRN deployment has been considered as a cost-effective solution to serve data-intensive UE inside public transportation vehicles [4].

In this article, we discuss the potential advantages as well as the implementation challenges of deploying MRNs. Compared to analog repeaters, advanced relay nodes (RNs) in LTE-Advanced systems can decode and forward data to UE. They can also detect or correct errors before forwarding the data to UE. Thus, the received signal qualities at the UE can be enhanced by using such RNs. Two types of advanced RNs, type 1 and type 2 RNs, have been defined by 3GPP. Type 1 RNs are non-transparent RNs that can form their own cells, and terminate all layer 2 and layer 3 communication protocols. Type 2 RNs are transparent RNs that replicate the cell ID of their donor eNB (DeNB). ${ }^{2}$ The FRN, which has been standardized in the LTEAdvanced system, is an inband half-duplex decode-and-forward (DF) type $1 \mathrm{RN}$, while the 
use of other types of RNs in LTE networks has been left for further investigation [5, Ch. 30]. Similarly, the MRN proposed in recent 3GPP studies is also an inband half-duplex DF type 1 $\mathrm{RN}$ that can form its own cell inside a vehicle [4]. Moreover, MRNs can be shared by several operators, a fact that can lower the cost of building the networks [6].

Initially, in 3 GPP studies, MRNs were designed to serve UE inside well isolated highspeed trains [4]. Recent studies show that MRNs can also effectively serve UE inside public transportation vehicles in general (buses, trams, etc.) $[7,8]$. There are several advantages of using MRNs. First, group handover (HO) can be performed by regarding the UE served by the same MRN as a group. Depending on the mobility management schemes, the signaling at the radio network side and probability of $\mathrm{HO}$ failure of the vehicular UE could be noticeably lowered [4, 9]. Second, since MRNs are not as limited by size and power as regular UE, they can better exploit various smart antenna techniques and advanced signal processing schemes. For example, in a train, several backhaul antennas can be interconnected to form a cooperative and coordinated relay system [10], which can strengthen the backhaul link by using antenna selection techniques. Moreover, by a proper placement of indoor and outdoor antennas, an MRN can circumvent the vehicular penetration loss (VPL) caused by a well isolated vehicle. Measurements show that the VPL can be as high as $25 \mathrm{~dB}$ in a minivan at the frequency of $2.4 \mathrm{GHz}$ [11], and higher VPLs are foreseeable in the vehicles of interest. Last but not least, compared to direct transmission from eNBs to in-vehicle UE, better propagation conditions - less shadowing and path loss, and higher line-of-sight (LOS) connection probabilities - can be expected for the backhaul connections between eNBs and MRNs.

Besides using MRNs, several other solutions using existing techniques have also been proposed in 3GPP to serve vehicular UE [4]. In this article, we first review the solutions based on the existing techniques to serve vehicular UE. Then we present the benefits of using dedicatedly deployed MRNs to serve UE inside public transportation vehicles. Simulation results show that MRNs can effectively serve vehicular UE. At the end of the article, we discuss the challenges and open issues as well as future research directions toward applying MRNs to practical communication systems.

\section{CuRRent SOlutions to Serve Vehicular UE}

Solutions based on existing system elements, such as optimizing the deployment of macrocells, or using layer 1 repeaters or $\mathrm{WiFi}$ access points (APs) to assist serving vehicular UE, have been discussed in 3GPP [4]. In this section, we briefly review the advantages and disadvantages of these solutions. All layer 1 repeaters, WiFi APs, and MRNs communicate with a macro eNB via radio interfaces, but MRNs also act as regular eNBs for the UE they serve, and can potentially support multiple radio access technologies.

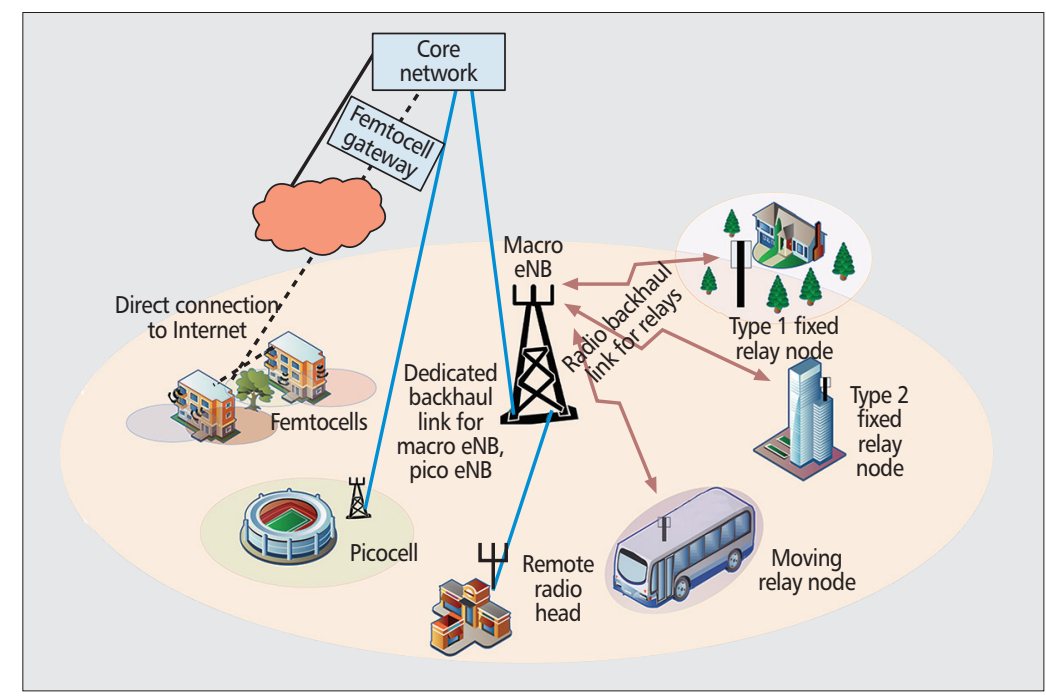

Figure 1. An overview of 3GPP heterogeneous and small cell networks.

\section{Dedicated Macro eNBs}

As the route of a public transportation vehicle is usually known, the coverage of macro eNBs can be tuned along the train lines or highways by using directive antennas. A trade-off between coverage and capacity can be addressed by employing an umbrella structure. More specifically, high-power eNBs can be used to extend coverage and handle control signaling with underlying low-power nodes to improve capacity. For instance, with CA, the primary component carrier (PCC) is transmitted by a high-power eNB, but the secondary component carriers (SCCs) can be transmitted by low-power nodes. ${ }^{3}$ By employing sophisticated cross-carrier scheduling schemes, most of the control signaling can be sent by the high-power eNBs, and the data can be transmitted by the low-power SCCs. If CA is not possible, the use of RRHs can be considered. Since RRHs share the same cell ID as the macro eNB, the closest RRH can serve the high speed vehicle without $\mathrm{HO}$ mechanisms being triggered.

However, the VPL, which is one of the major challenges faced by vehicular UE, cannot be reduced by this solution. Site acquisition, deployment, and maintenance are also challenges for operators, especially for low-power nodes, due to the requirements for power supply, dedicated backhaul connection, and so on. Moreover, this solution cannot easily be extended to urban scenarios.

\section{LAYER 1 REPEATERS}

Layer 1 repeaters are low-cost analog repeaters, as little signal processing is required compared to the advanced RNs in LTE. They amplify and forward signals in a given frequency band, and they can possibly work in a full- duplex mode on the vehicles of interest, as the vehicles can sufficiently isolate the transmit and receive chains. This is an advantage compared to the half-duplex FRNs standardized in LTE. ${ }^{4}$ Moreover, layer 1 repeaters can overcome the VPL caused by well isolated vehicles, and thereby lower the UE transmit power.

However, there are several issues with layer 1
${ }^{3}$ With CA, up to four SCCs can be aggregated with a PCC, and each of the component carriers defines its own serving cell. Different component carriers may be configured to provide different coverage. The configuration of $P C C$ and SCCs is terminal-specific: depending on the system load, UE served by the same macro eNB can have different $P C C$ and SCCs assignments [5, Ch. 28]

${ }^{4} A$ half-duplex $R N$ can transmit and receive in both directions but not simultaneously, whereas a full-duplex $R N$ provides connection in both directions at the same time. To achieve full duplexing, good isolation between transmit and receive chains is required due to self-interference, such as using frequency-division duplexing (FDD). 


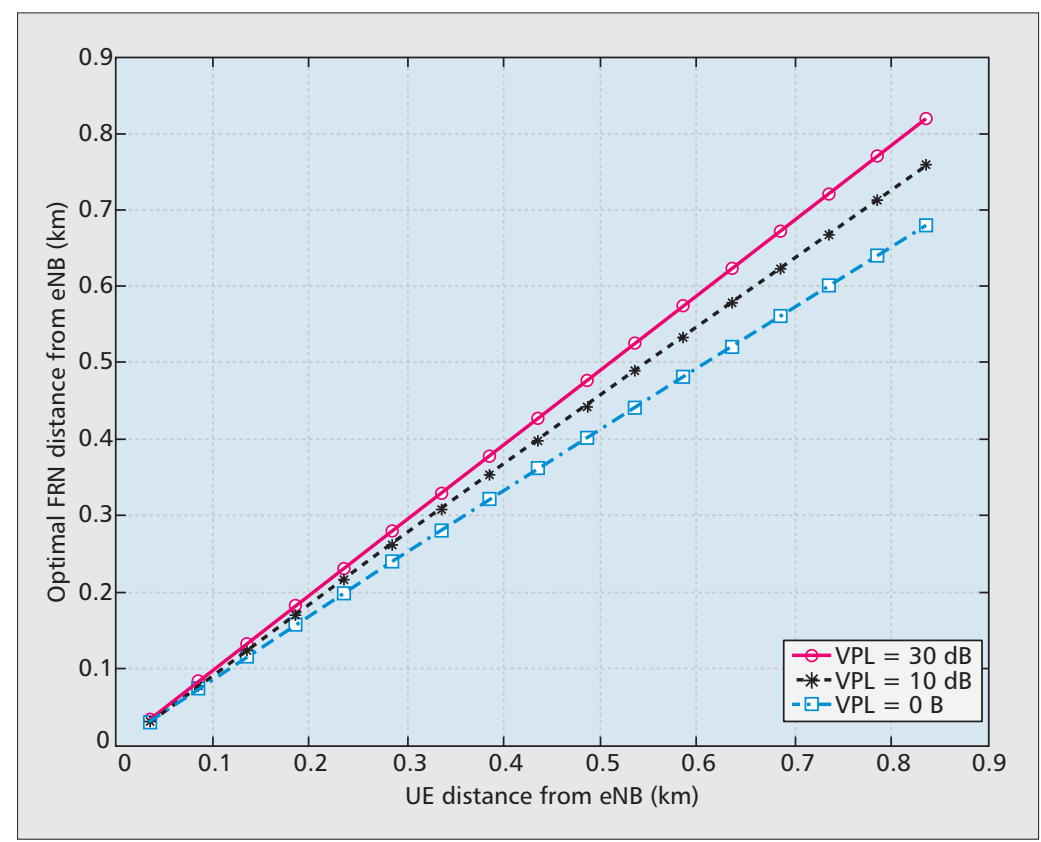

Figure 2. Optimal FRN position when UE position is known.

repeaters. Compared to advanced DF RNs, the signal-to-interference-plus-noise ratio (SINR) cannot be improved at layer 1 repeaters, since they amplify and forward both the received desired and interference signals. ${ }^{5}$ Furthermore, since layer 1 repeaters are less controlled by the core network than the advanced RNs, the $\mathrm{HO}$ of vehicular UE between eNBs still needs to be performed individually, and advanced interference management schemes cannot be integrated in them. Thus, layer 1 repeaters may bring more interference into the system.

\section{LTE FOR BACKHAUL, WIFI FOR ACCESS}

Using WiFi to provide Internet access to data users on board is fairly common, as most smart phones and laptops are WiFi-capable. Similar to layer 1 repeaters, wireless backhaul nodes can be placed on the roof of a vehicle with antennas mounted outside communicating with the cellular network, while providing data access to vehicular users with WiFi APs. Backhaul links can be full duplex. WiFi is used for data connections, and regular voice calls can be handled by the cellular network. Hence, VPL can be circumvented, and group $\mathrm{HO}$ can be used for data users. Furthermore, WiFi-only devices can also use the cellular network, which may bring extra income to service providers.

However, using WiFi technology to provide UE with a seamless experience in the current cellular network is challenging. Although the 802.11x extension of the WiFi standard enables roaming for WiFi devices, functions such as charging, authentication, and security still require software and hardware upgrades for the WiFi module to access the universal subscriber identity module (USIM) in the device [12]. Moreover, although the 802.11e amendment of the WiFi standard has quality of service (QoS) support, it requires that all the APs are controlled by the same central unit [13]. As WiFi networks operate on the open industrial, scientific and medical radio (ISM) bands, the interference in these radio bands cannot be coordinated in the same manner as in the dedicated frequency bands owned by operators. Thus, it is difficult for the operators to offer similar QoS as in their own cellular networks.

\section{Dedicated MRN DePLOYMENT}

In this section, we discuss the deployment of dedicated MRNs to serve UE inside public transportation vehicles. To motivate the use of MRNs to serve vehicular users, we consider a simple example. In a noise limited system, assume that the position of a vehicular UE device is known, and we are interested in finding the optimal location for an FRN to minimize the end-to-end outage probability (OP). This setup was studied in [14], and Fig. 2 plots the optimal FRN position as a function of VPL by using a typical urban propagation model. The plot indicates that if we know the communication is affected by high VPL, the best solution is to place an FRN as close as possible to the vehicle to compensate for the VPL. In practice, as the positions of vehicles are not known beforehand, the use of MRN is more economical and applicable to serve vehicular UE.

In an interference limited scenario, the MRN can bring additional benefits. As shown in $[8$, 10], MRNs can operate at much lower transmit power compared to macro eNBs or other lowpower nodes, but still achieve similar or even better performance. As shown in [8], when the VPL is $30 \mathrm{~dB}$, a half-duplex MRN can lower the end-to-end OP by 65 percent on average compared to direct transmission at vehicular UE, and even better performance is expected from a full-duplex MRN.

In order to understand the performance of an MRN serving vehicular UE in a more practical setup, we resort to system-level simulations. A semi-static system simulator is used, and the simulated network consists of 19 cells. On average we drop 25 macro UE devices per cell, and one randomly located bus with five UE devices onboard moving through the cells. A system bandwidth of $10 \mathrm{MHz}$, corresponding to 50 physical resource blocks (PRBs), is considered. VPL is varied between 0 and $30 \mathrm{~dB}$, and detailed simulation parameters are given in Table 1. Both half-duplex and full-duplex MRNs are considered, and the performance is compared with the baseline case(i.e., vehicular UE devices are served directly by macro eNBs). The half-duplex MRN assisted transmission is implemented in such a way that, in the first transmission time interval (TTI), the DeNB sends to the MRN (via backhaul link), and in the following TTI, the MRN communicates to the UE devices in the vehicle; eNBs communicate to macro UE devices in both TTIs.

Figure 3 shows the downlink performance of macro UE and vehicular UE separately, with varying VPL. The cumulative distribution function (cdf) of the UE throughput is calculated. The results in Fig. 3 are shown as 95 percent UE throughput (horizontal axis) vs. 5 percent throughput (vertical axis, corresponding cell 
edge performance). The throughput of vehicular UE served by a half-duplex MRN is roughly halved compared to the full-duplex MRN case due to the half-duplex loss. Nevertheless, as VPL increases, the cell edge performance of the vehicular UE served by an MRN, for both the half-duplex and full-duplex cases, is greatly improved compared to the baseline case. The performance improvement is due to two reasons:

- Relaying circumvents VPL.

- The MRN is installed on the roof of the vehicle, and thereby better propagation conditions (i.e., less shadowing and path loss) are experienced by the backhaul link compared to the direct link from macro eNBs to vehicular UE.

Regarding the macro UE, the duplex scheme employed by the MRN (square for full-duplex MRN case and diamond when half-duplex MRN is used) has no significant impact on the 95 percent UE throughput. However, there is a noticeable decrease of the 5 percent throughput for the macro UE when a full-duplex MRN is used to serve vehicular UE compared to the halfduplex MRN case. This is because with a halfduplex MRN, half of the time there is no backhaul link connection, and during these TTIs all PRBs are instead used to serve macro UE. Moreover, when the backhaul link of the MRN is active in one cell, it generates interference to the macro UE in other cells. We also notice that there are no significant impacts of different VPLs on the performance of macro UE.

The results show a drastic increase in cell edge performance for the vehicular UE. In this example, the number of vehicular UE devices is quite small compared to macro UE devices. With a higher number of vehicular UE devices, the performance would probably be affected since increasing the number of PRBs for the backhaul link would also decrease the macro UE performance. Nevertheless, we can conclude that the use of MRNs deployed on the vehicle improves performance of the vehicular UE, especially at the cell edge.

\section{Challenges And Open Issues}

The potential of using MRNs to improve the QoS and throughput of vehicular UE is outlined in previous sections, but there are also challenges. In this section, we briefly discuss the challenges of deploying MRNs and possible solutions.

From the scenarios studied earlier, we can identify that the backhaul link is the capacity bottleneck. As the UE speed goes up, the rapid variations of mobile channels combined with feedback delays reduce the accuracy of the channel state information (CSI) at eNBs. The CSI feedback inaccuracy at the eNB limits the use of advanced MIMO transmission schemes employed in LTE systems to further increase the throughput of the backhaul link, as adaptive transmit beamforming, link adaptation, and channel-aware scheduling would be severely compromised. In FDD LTE systems, the CSI at the eNB would be outdated by at least $5 \mathrm{~ms}$ due to channel estimation delays, the frame structure, and feedback delays. Although LTE-

\begin{tabular}{|c|c|}
\hline Parameter & Value \\
\hline Carrier frequency & $2.6 \mathrm{GHz}$ \\
\hline System bandwidth & $10 \mathrm{MHz}$ \\
\hline Number of PRBs & 50 \\
\hline Number of cells & 19 \\
\hline Intersite distance (ISD) & $500 \mathrm{~m}$ \\
\hline Duplex scheme & Full-duplex (FDD) and half-duplex \\
\hline Relaying type & In-band, decode-and-forward \\
\hline Vehicle position & Random, uniform distribution \\
\hline Number of vehicular UE devices & 5 \\
\hline Number of macro UE devices/cell & 25 (average) \\
\hline Number of macro UE devices & 475 (total) \\
\hline UE position & Random, uniform distribution \\
\hline VPL & $0,10,20,30 \mathrm{~dB}$ \\
\hline Number of receive antennas & 2 (RN, UE) \\
\hline PRB allocation principle & Resource (PRB) fair \\
\hline Frequency domain allocation & Random \\
\hline eNB transmission power & $46 \mathrm{dBm}$ \\
\hline MRN transmission power & $10 \mathrm{dBm}$ \\
\hline Shadowing standard deviation eNB-UE & $8 \mathrm{~dB}$ \\
\hline Shadowing standard deviation eNB-MRN & $4 \mathrm{~dB}$ \\
\hline Receiver noise figure & $8 \mathrm{~dB}$ \\
\hline Traffic model & Full buffer \\
\hline
\end{tabular}

Table 1. Simulation parameters.

Advanced systems offer several diversity-based schemes for high-mobility UE, more resources are then required to be allocated to these UE devices. This lowers the spectral efficiency of the time-varying MRN backhaul link compared to that of an FRN in a similar position.

Using channel predictors, such as Kalman or Wiener predictors, could potentially enable the use of adaptive transmit techniques at vehicular velocities, as they can predict fading channels in space $0.2-0.3$ wavelengths ahead with adequate accuracy. This means that $5 \mathrm{~ms}$ prediction horizons could be attained for low vehicular velocities at $900 \mathrm{MHz}$ carriers but not for frequency above $1.5 \mathrm{GHz}{ }^{6}$ The scenario with an antenna or antenna array on the roof of a fast-moving bus or tram provides an opportunity as well as a challenge. One innovation is the use of a "predictor antenna" [15]. The vehicle velocity and
${ }^{6} A$ vehicle at a speed of $50 \mathrm{~km} / \mathrm{h}$ moves $0.0694 \mathrm{~m}$ in $5 \mathrm{~ms}$, which is a bit more than 0.2 wavelengths at $900 \mathrm{MHz}$. 


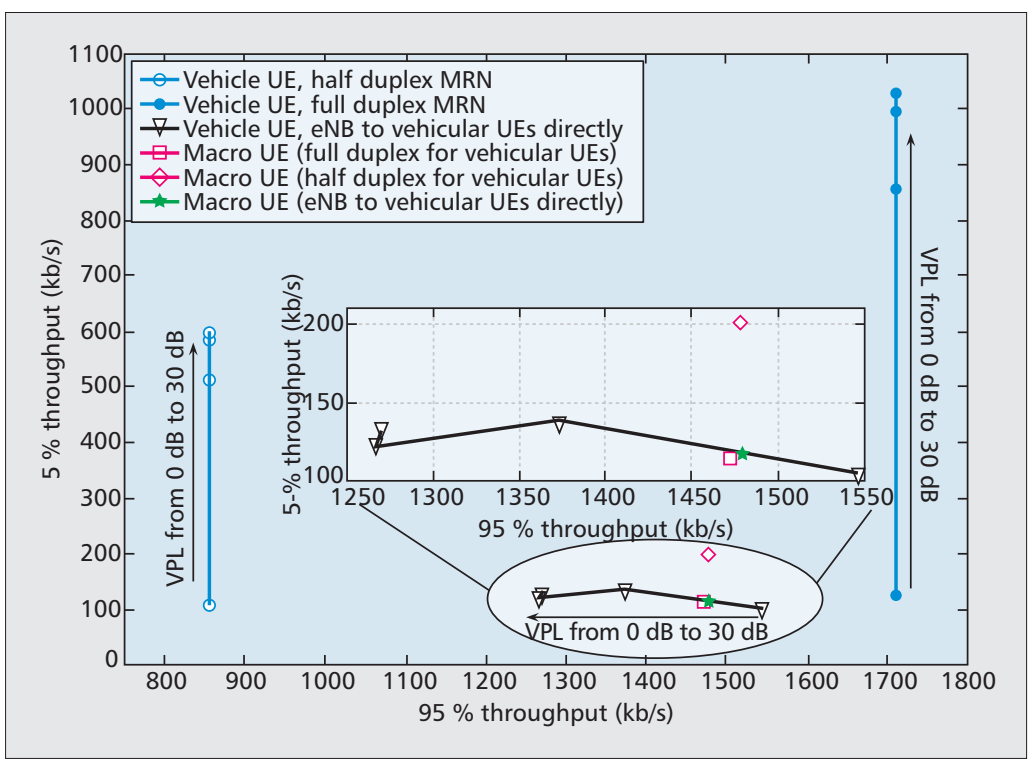

Figure 3. Downlink performance of MRN assisted transmission.

direction would be relatively constant over the required $5 \mathrm{~ms}$ prediction horizon. Channel predictions could then be obtained by the predictor antenna located some distance directly in front of the antennas used for transmission, as shown in Fig. 4. Channel estimates from this antenna could be used to predict the channel experienced by the rearward antenna a little later when it has moved to this position. We would avoid the need to extrapolate in time from the measurements.

Preliminary results based on measurement campaigns done in a $20 \mathrm{MHz}$ orthogonal frequency-division multiplexing (OFDM) system in $2.68 \mathrm{GHz}$ downlinks showed that using a predictor antenna can significantly improve the CSI feedback accuracy at a vehicular velocity [15]. A maximal normalized correlation above 0.95 was reported between the signal from the predictor antenna and the signal from the antenna behind it mounted on the vehicle roof, with antenna distances (prediction horizons) of up to three wavelengths, in both LOS and non-LOS (NLOS) conditions. This suggests that a simple predictor that uses delayed channel estimates from a predictor antenna can provide useful prediction accuracy at vehicular velocities. Thus, it opens up the possibility of using the most advanced MIMO techniques as well as link adaptation to increase the throughput over the backhaul links of MRNs.

Another challenge in the deployment of MRNs is from the aspect of mobility management. This includes the HO of MRNs between different DeNBs, and HO of UE between MRNs and eNBs. Previous studies showed that by performing group $\mathrm{HO}$, the $\mathrm{HO}$ failure probabilities of vehicular UE can be lowered significantly by using MRNs [9]. However, as the LTE system today has no mobility support for MRNs, modification of the current system architecture is needed to provide efficient yet reliable mobility management for MRNs [4, 7].

Figure 5 shows the current FRN architecture employed by the LTE system. A DeNB acts as a proxy, and hides an FRN from mobility management entities (MMEs) and various gateways serving the UE. As the time required for FRN attachment and configuring the proxy functionality at a DeNB is on the order of minutes $[5, \mathrm{Ch}$. 30], the same FRN system architecture cannot be directly adapted for MRNs. On the radio network side, by using MRNs, the signaling overhead can be reduced by performing MRN HO instead of individual UE HO. However, on the core network side, the data connection of each UE device still needs to be handled individually at the MME [4]. Although this is not required to be done at the same time as the HO of UE, depending on the network topologies and whether the target DeNB is served by the same MME or MME pool, the disturbance caused by the HO may be very large [7]. To support MRN mobility, several solutions either based on modifications of the current FRN architecture or using an entirely new architecture have been discussed, and various architectures have been compared in $[4,7]$. An overall comparison of different mobility management architectures is given in [4, Table 6.1], and detailed HO delays are analyzed in [7]. Moreover, in a HetSNets deployment scenario, there could potentially be problems created by a large group of vehicular UE devices that are handed over into a fixed small cell, thereby disturbing its resource allocation. But we are already facing an even worse variant of this problem today, when lots of individual vehicular UE devices move through a fixed network with small cells. Last but not least, how to avoid UE devices close to but outside the vehicles wrongly attaching to the MRNs also needs consideration.

Furthermore, new challenges regarding interference management arise due to the use of MRNs. As the distance between an MRN and the vehicular UE served by it is very short, the MRN and the vehicular UE can communicate with each other using very low power. In addition, the VPL can further help to dampen the signal of the MRN access link that propagates out from the vehicle. Thus, compared to direct transmission, the use of MRNs generates less interference from the access link, for both downlink and uplink, to UE outside the vehicles. This is appreciated in a densely deployed urban scenario where link availabilities are usually dependent on interference rather than coverage. For the backhaul link, however, the problem becomes complicated, as interference is expected both between different MRN backhaul links, and between MRN backhaul links and macro UE. The use of predictor antennas can improve CSI accuracy to enable the use of advanced interference avoidance and cancellation schemes for the backhaul links. Nevertheless, whether enhancements on the current intercell interference coordination (ICIC) framework in LTE are needed to support the use of MRNs still requires further investigation.

\section{DISCUSSION AND FUTURE DIRECTIONS}

In this article, we present the benefits and challenges of using dedicated MRNs to serve vehicular UE. Compared to serving vehicular UE directly from macro eNBs, MRNs can increase 
the performance of the UE inside the vehicle significantly, especially at the cell edge. This is because MRNs can effectively reduce VPL, and create better propagation conditions for the backhaul links compared to the direct links between macro eNBs and vehicular UE. As the backhaul connections of the MRNs become the bottleneck, we present the idea of using prediction antennas to increase the accuracy of the CSI feedback for the backhaul links. This improves the performance of channel-dependent scheduling and enables the use of spatial processing for the backhaul links of MRNs at high vehicular velocities.

However, there are several challenges faced by using MRNs, both technically and economically. The use of MRNs brings new tasks regarding mobility and interference management to the community. Several mobility management solutions have been proposed with initial evaluation results, but which one should be adopted in the future system still requires further investigation. Moreover, whether new ICIC enhancements are needed to support the use of MRNs is still an open issue. From the business logic point of view, on one hand, using MRN can significantly improve the user experience, and thereby increase the loyalty of customers. On the other hand, it will increase the operation costs of operators. Public transportation companies could potentially share these costs. Also, novel context or locationawareness-based services targeting the needs of travelers and commuters could evolve into new business models in the near future. Further investigations are needed to answer these broad questions. Nevertheless, the use of MRNs has great potential as an efficient solution to the ever growing ubiquitous demand for high-quality mobile data services by vehicular users.

\section{ACKNOWLEDGMENT}

The authors would like to thank the Swedish Research Council (VR) and the EU FP7 for providing the research funds. We also appreciate the contributions from our colleagues in the EU FP7 project INFSO-ICT-247223 ARTIST4G.

\section{REFERENCES}

[1] A. Ghosh et al., "LTE-Advanced: Next-Generation Wireless Broadband Technology," IEEE Trans. Wireless Commun., vol. 17, no. 3, June 2010, pp. 10-22.

[2] A. Damnjanovic et al., "A Survey on 3GPP Heterogeneous Networks," IEEE Trans. Wireless Commun., vol. 18, no. 3, June 2011, pp. 10-21.

[3] Cisco white paper, "Cisco Visual Networking Index: Global Mobile Data Traffic Forecast Update, 2011-2016," tech. rep., http://www.cisco.com/, accessed Nov. 20th, 2012.

[4] 3GPP TR 36.836, "Technical Specification Group Radio Access Network; Mobile Relay for Evolved Universal Terrestrial Radio Access (E-UTRA)," tech. rep., http://www.3gpp.org/, accessed Nov. 20, 2012.

[5] S. Sesia, I. Toufik, and M. Baker, LTE - The UMTS Long Term Evolution: From Theory to Practice, 2 nd ed., Wiley, 2011.

[6] 3GPP TR 22.951, "Service Aspects and Requirements for Network Sharing," tech. rep., Sept. 2012, http://www.3gpp.org/, accessed Nov. 20, 2012.

[7] M. Khanfouci, Y. Sui, A. Papadogiannis, and M. Färber, "D3.5-c Moving Relays and Mobility Aspects," tech. rep., Artist4G deliverable, May. 2012, https://ictartist4g.eu/projet/deliverables accessed Feb. 14, 2013.

[8] Y. Sui et al., "Performance Comparison of Fixed and Moving Relays under Co-Channel Interference," Proc. IEEE GLOBECOM Wksps., 2012.

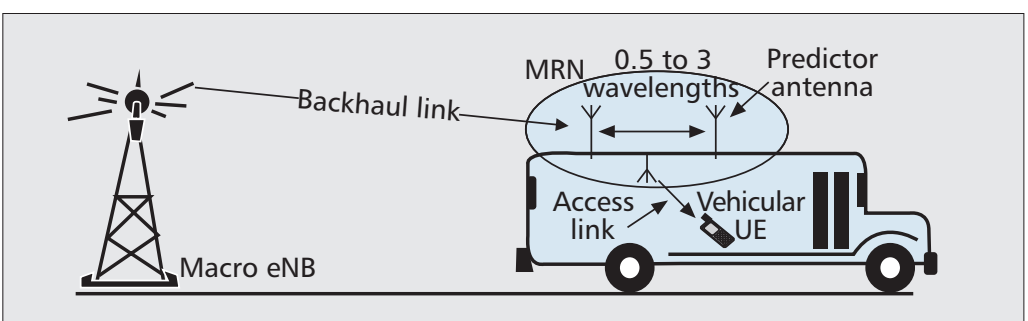

Figure 4. An illustration of deploying an MRN on top of a public transportation vehicle.

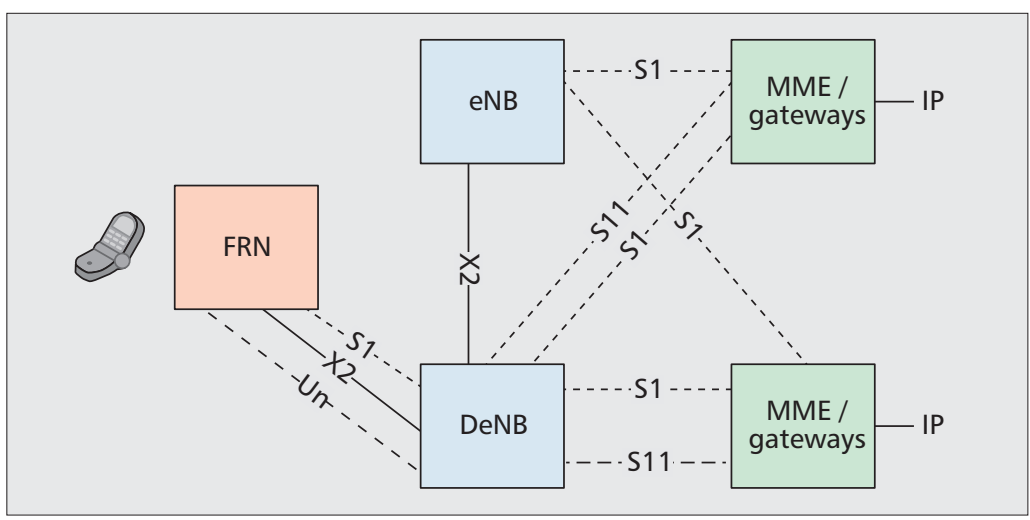

Figure 5. FRN system architecture in LTE release 10.

[9] W. Li et al., "Performance Evaluation and Analysis on Group Mobility of Mobile Relay for LTE-Advanced System," Proc. IEEE VTC 2012-Fall.

[10] V. V. Phan et al., "Providing Enhanced Cellular Coverage in Public Transportation with Smart Relay Systems," Proc. IEEE VNC, 2010.

[11] E. Tanghe et al., "Evaluation of Vehicle Penetration Loss at Wireless Communication Frequencies," IEEE Trans. Vehic. Tech., vol. 57, no. 4, July 2008, pp. 2036-41.

[12] 3GPP Std. TS 23.234, "3GPP System to Wireless Local Area Network (WLAN) Interworking; System Description," Sept. 2012, http://www.3gpp.org/, accessed Nov. 20, 2012.

[13] IEEE 802.11 WG, "Wireless LAN Medium Access Control (MAC) and Physical Layer (PHY) Specifications. Amendment 8: Medium Access Control (MAC) Quality of Service Enhancements," IEEE 802.11e, Nov. 2005.

[14] Y. Sui, A. Papadogiannis, and T. Svensson, "The Potential of Moving Relays - A Performance Analysis," Proc. IEEE VTC 2012-Spring.

[15] M. Sternad et al., "Using 'Predictor Antennas' for Long-Range Prediction of Fast Fading for Moving Relays," Proc. IEEE WCNC, 2012.

\section{BIOGRAPHIES}

YUTAO SUI [S'11] (sui.yutao@chalmers.se) received his B.E. degree in electronic engineering and B.A. degree in English from Yanbian University, Yanji, China, in 2006, and his M.Sc. degree in wireless communications from Lund University, Sweden, in 2009. He is currently a Ph.D. candidate in the Department of Signals and Systems at Chalmers University of Technology, Gothenburg, Sweden. He concentrates his research on heterogeneous and small cell networks, especially moving relays for vehicular user communications.

JAAKKO VIHRIÄLÄ (jaakko.vihriala@nsn.com) received his M.Sc. degree from the University of Oulu, Finland, in 1995. He joined Nokia in 1993, and is currently with Nokia Siemens Networks, Oulu. He has been working on DSP algorithm design and implementation, WCDMA receiver design, wireless relaying, and heterogeneous networks.

AgISILAOS PAPAdogIANNIS [S'07, M'10] (apapadogiannis@ gmail.com) received his $M$.Eng. degree in electrical and computer engineering from the University of Patras, Greece, in 2005, his M.Sc. (with distinction) degree in com- 
munications engineering from the University of York, United Kingdom, in 2006, and his Ph.D. degree in electronics and communications from TELECOM ParisTech, Paris, France, in 2009. From 2006 to 2009 he was a research engineer at Orange Labs, Paris, working on 4G communications. The focus of his work was on coordinated multipoint (CoMP) and relaying techniques with affordable overheads in relation to the 3GPP LTE-Advanced standards. From April 2010 to August 2011 he was a research associate at the Department of Electronics, University of York, working on technologies beyond 4G. From August 2011 to February 2013 he was a postdoctoral fellow at the Department of Signals and Systems, Chalmers University of Technology, where he conducted teaching, research, and research supervision in the areas of future user-centric and energy-efficient networks. He has participated in several European (FP7 METIS, FP7 ARTIST4G, FP7 BuNGee, CELTIC WINNER+), nationally funded (VR Dynamic Multipoint Wireless Transmission, VINNOVA MAGIC, VINNOVA MORE4G, RCUK UC4G), and industrial research projects (4GRADIO, PRICE) in the general area of future wireless systems. He holds two international patents, and has authored a book and numerous high impact technical papers.

MikAel Sternad [S'83, M'88, SM'90] (mikael.sternad@signal.uu.se) is a professor of automatic control at Uppsala University and received a Ph.D. degree from Uppsala University in the same field in 1987. With a background in robust and adaptive estimation, control, and filtering, he led the national Swedish project Wireless IP and is at present leading the Dynamic Multipoint Wireless Access framework project funded by the Swedish Research Council. He has participated in the EU WINNER, WINNER II, and ARTIST4G Integrated Projects, contributing to channel prediction and estimation, adaptive transmission, design of robust linear precoders, deployment concepts, multiple access techniques/concepts, MAC layer design, and overall system design. Channel prediction has been one focus area of research for 15 years. He is also involved in audio and acoustic MIMO signal processing, room compensation, and sound field control. He co-founded and was chairman (2001-2005) of Dirac Research, a company in this area. He has authored over 130 papers, six book chapters, and 10 patents.

WEI YANG [S'09] (ywei@chalmers.se) received his B.E. degree in communication engineering and M.E. degree in communication and information systems from Beijing University of Posts and Telecommunications, China, in 2008 and 2011, respectively. He is currently pursuing a Ph.D. degree in electrical engineering at Chalmers University of Technology. From July to August 2012, he was a visiting student at the Laboratory for Information and Decision Systems, Massachusetts Institute of Technology, Cambridge. He is the recipient of a Student Paper Award at the 2012 IEEE International Symposium on Information Theory, Cambridge, Massachusetts. His research interests are in the areas of information and communication theory.

TOMMY SVENSSON [S'98, M'03, SM'10] (tommy.svensson@ chalmers.se) is an associate professor in communication systems at Chalmers University of Technology, where he is leading the research on air interface and microwave backhaul networking technologies for future wireless systems. He received a $\mathrm{Ph}$.D. in information theory from Chalmers in 2003, and has worked at Ericsson AB with core networks, radio access networks, and microwave transmission products. He participated in the European collaborative research projects WINNER I, II, WINNER+, and ARTIST4G, which have contributed to the 3GPP LTE standards, and he is currently active in the EU FP7 METIS project targeting solutions for 2020 . His main research interests are in design and analysis of physical layer algorithms, multiple access, resource allocation, cooperative systems, and moving relays/cells. He has co-authored two books and more than 70 journal and conference papers. $\mathrm{He}$ is Chairman of the IEEE Sweden VT/COM/IT chapter, and coordinator of the Communication Engineering Master's Program at Chalmers. 